\title{
Brief Resilience Scale
}

National Cancer Institute

\section{Source}

National Cancer Institute. Brief Resilience Scale. NCI Thesaurus. Code C153304.

A scale developed by Smith, et al, designed to assess an individual's ability to recover from stressful circumstances. 